\title{
Time resolved scattering relaxation mechanisms of microcavity polaritons
}

\author{
F. Chaves and F. J. Rodríguez* \\ Departamento de Física, Universidad de Los Andes, A.A. 4976, Bogotá D.C., Colombia.
}

\begin{abstract}
We study the polariton relaxation dynamics for different scattering mechanisms as: Phonon and electron scattering procesess. The relaxation polariton is obtained at very short times by solving the Boltzman equation. Instead of the well-known relaxation process by phonons, we show that the bottleneck effect relaxes to the ground state more efficiently at low pump power intensity when the electron relaxation process is included. In this way, we clearly demonstrate that different relaxation times exist, for which any of these two mechanism is more efficient to relax the polariton population to the ground state.
\end{abstract}

\section{INTRODUCTION}

Many interesting and unusual dynamical quantum many-body phenomena have been the subject of an extended study. In particular, using ultrafast laser technology, the dynamics of many-body processes can be understood from a fundamental point of view. Extended reports in the literature suggest that due to the strong coupling between radiation-matter, signatures of a possible condensation of bosonics particles can be detected. One of the most spectacular and still controversial effects is the possibility to get Bose Eintein Condensation of polaritons in quantum wells embedded in semiconductor microcavities. The accumulation in the ground state of polaritons has been suggested by different experiments.1.2.3.4 and predicted theoretically by 5.6.7. $^{\text {. B }}$ By illuminating the sample with lasers of different frequencies, it is possible to get a matter-wave amplification without requiring a coherent pumplaser ${ }^{8.9}$ However, one of the main difficulties to obtain a Bose condensation of polaritons has been the relaxation of the bottleneck which hinders that excitons relax quickly to the optically active lower energy states. This bottleneck effect, can be relaxed if the pump-laser is increased or if different relaxation scattering mechanisms become more efficient to allow that the population relaxes to the ground state $\frac{10.11}{\text {. }}$.

A comprehensive study of the main mechanisms of polaritonic relaxation, comprises electron, phonon and polaritonpolariton scattering. In the stationary limit, Malpuech et al11, have shown results for each of the above mentioned scattering mechanism for different pump laser intensities. As can be experimentally observed, for some range of carrier densities, where the strong-coupling remains, the polariton-polariton relaxation is very inneficient $\frac{12.13}{13}$ to overcome the bottleneck. However, in semiconductor microcavities, under non-resonant excitation, acoustic phonons provide an effective channel for polaritons relaxation ${ }^{14.15}$. Moreover, recent experiments ${ }^{1.16}$ have shown the importance of electron-polariton scattering at densities where the electron-electron interaction are insufficiently effective due to the their fast relaxation. This two relaxation mechanisms could be the most relevant to relax the huge population at the bottleneck. In spite of the wide amount of experimental and theoretical 17.18 studies on this subject that has appeared, theoretical and detailed calculations as a function of time are very few and consider only one type of relaxation processes. The case in which both electron- and phonon-polariton interactions are present has only been studied recently in the stationary limit $\frac{1}{\underline{\underline{1}}}$ either for high laser power excitations or weak detuning regimes. In our opinion one of the important questions in the area of polariton relaxation dynamics is the effect of the different relaxation mechanisms in the transient time range. It has been recently suggested that in the transient time and in an isotropio $\frac{15}{15}$ system, an optimal relaxation bottleneck can be achieved only by increasing the power laser excitation. In this paper, we show that this result becomes ambiguous and, as is well known, in the stationary limit, is partially true in a restricted range of power excitation.

We report in this work on the dynamics of polaritons continuously excited by a laser and including both electron- and acoustic-phonon scattering process. This introduces quantitative and qualitative different features for the dynamics following the resonant excitation. The purpose of the present paper is to shed light about which is the more efficient relaxation mechanism for polaritons for a given pump laser power and for a finite electron concentration, as a function of time and momentum. The main result is that electron-polariton (el-pol) relaxation is the most efficient mechanism, when compared with the phonon-polariton (ph-pol) relaxation. The road to studying the contribution of different scattering mechanisms has lead to use one of the more accepted models based on solving the Boltzmann equation, because in this way it is very easy to include different scattering mechanisms and capture the essential physics involved in the polaritonic relaxation process.

\footnotetext{
* Email address: frodrigu@uniandes.edu.co
} 
The paper is structured as follows: Section ПA describes the Hamiltonian interaction between excitons and photons. Sec IB describes the rate equation and the scattering process including both phonon and electron scattering. Section III shows explicitly how our numerical simulation gives the temporal range in which either scattering mechanisms are more important. Finally we present discussions and conclusions of our results and establish the comparison with another theoretical result.

\section{THEORETICAL MODEL}

We describe first in this section the model that we use in order to describe the polariton relaxation. We assume through this work that the polaritons interact with phonons through a deformed and not a piezoelectric potential.

\section{A. Interacting Hamiltonian}

The usual experimental set-ups consider a quantum well embedded in a microcavity which allows a stronger coupling between the two dimensional excitons and the confined light. The excitons are generated by pumping at lower excitation and an attractive Coulomb interaction arises, showing an spectrum similar to the hydrogen atom. The spectrum of the bound exciton states in a quasi-two dimensional system is given by

$$
\omega_{e}(\vec{k})=-\omega_{e}(0)+\frac{k^{2}}{2 M}
$$

where $M=m_{e}+m_{h}, \omega_{e}(0)=\frac{m_{x} e^{4}}{2 \epsilon_{s}^{2}}, m_{x}=m_{e} m_{h} / M_{x}, \epsilon_{s}$ is the effective dielectric semiconductor constant and $m_{e(h)}$ represents the effective electron (hole) mass of the carriers in the semiconductor, $(\vec{k})$ the in-plane momentum vector $(\hbar=1)$. In this way the total Hamiltonian can be written as:

$$
H=\omega_{\text {cav }}(\vec{k}) c_{\vec{k}}^{\dagger} c_{\vec{k}}+\omega_{e}(\vec{k}) e_{\vec{k}}^{\dagger} e_{\vec{k}}+\Omega_{0}\left(c_{\vec{k}}^{\dagger} e_{\vec{k}}+e_{\vec{k}}^{\dagger} c_{\vec{k}}\right)
$$

where $\omega_{\text {cav }}(\vec{k})=\frac{c}{n} \sqrt{k^{2}+\frac{\left(n \omega_{c}\right)^{2}}{c^{2}}}$, is the resonant cavity energy, $\omega_{e}(\vec{k})=\omega_{e}(0)+\frac{k^{2}}{2 M}$ is the exciton dispersion enrgy, $\omega_{c}=\frac{\pi c}{n L}, n$ is the refraction index of the semiconductor and $L$ the effective well width. The photon(exciton) creation operators are given by $c_{\vec{k}}^{\dagger}\left(e_{\vec{k}}^{\dagger}\right)$ and $\Omega_{0}$ is the coupling energy between the excitons and photons. By using a linear transformation the exciton-photon interaction Hamiltonian can be diagonalized which leads to new quasiparticles, called excitonic-polaritons ${ }^{19}$. This new quasiparticles arise by the mixing of the exciton and photon states. It is well known that at very low excitonic densities, the excitons follow Bose commutation relations, given that the excitons can be considered as the excited state of a N-particle system, in such a way that the excitons can be considered as bosonic particles. The Hamiltonian can be diagonalized to obtain the well known branches of the polaritons dispersion relation 19

$$
E_{L}^{U}(k)=\frac{\omega_{c a v}(\vec{k})+\omega_{e}(\vec{k})}{2} \pm \sqrt{\Omega_{0}^{2}+\frac{\Delta^{2}(\vec{k})}{4}}
$$

$\Delta(\vec{k})=\omega_{\text {cav }}(\vec{k})-\omega_{e}(\vec{k})$ represents the detuning between the exciton and cavity energies. For negative detunings $(k=0)$ a resonance condition can be achieved for higher in plane wave vectors.

\section{B. Phonon and electron relaxation mechanisms}

In order to describe better the experimentalist results ${ }^{10}$, we describe in detail the two most important relaxation mechanisms of polaritons to the lowest energy states for lower power excitations: (1) Acoustic phonon relaxation and (ii) Electron relaxation. Using the Boltzmann equation, the polariton relaxation rate is given by the following master equation:

$$
\frac{d p_{k}^{i}}{d t}=G_{\vec{k}}^{i}(t)-\frac{p_{k}^{i}}{\tau_{\vec{k}}^{i}}+\left(p_{\vec{k}}^{i}+1\right) \sum_{j, \vec{k}^{\prime}} W_{\vec{k}^{\prime}, \vec{k}}^{j, i} p_{\vec{k}^{\prime}}^{j}-p_{\vec{k}}^{i} \sum_{j, \vec{k}^{\prime}} W_{\vec{k}, \vec{k}^{\prime}}^{i, j}\left(p_{\vec{k}^{\prime}}^{j}+1\right)
$$


where $p_{\vec{k}}^{i}$ is the polariton distribution of the $i^{t h}$ branch, $G_{k}^{i}(t)=G_{0} T h\left(\frac{t}{T}\right)$ (in the following $T=20 p s$ ) is the continuous optical pumping generation, $\tau_{\vec{k}}^{i}$ is the time recombination rate. Due to the radiative and non radiative character of the polaritons, the radiative lifetime is a function of $k$. We have used the same values as in Ref $\underline{\underline{15}}$. The total scattering rate (phonons + electrons) is denoted by $W_{\vec{k}, \vec{k}^{\prime}}^{i, j}=W_{\vec{k}, \vec{k}^{\prime}}^{i, j(p h)}+W_{\vec{k}, \vec{k}^{\prime}}^{i, j(e)}$ and describes the transitions between two polariton states. It is worth pointing out that the algebraic properties of the above equation guarantee that its solution will consist of a linear combination of terms with a decaying exponential time-dependence, and will always show a stable approach to some steady state and it is appropriate for the description of the dynamics for low-density excitation.

-Acoustic phonon-polariton relaxation: For resonantly or non-resonant created excitons, the transition rates between two polaritonic states are proportional to the matrix elements between exciton states weighted by the respective exciton content of the polariton states.

$$
\begin{array}{r}
W_{\vec{k}, \vec{k}^{\prime}}^{i, j,(p h)}=2 \pi \sum_{q_{z}}\left|M_{p h}^{i, j}\left(\vec{k}-\vec{k}^{\prime}\right)\right|^{2}\left(n\left(\Omega_{\vec{q}, q_{z}}\right) \pm \frac{1}{2}+\frac{1}{2}\right) \\
\delta\left(E_{j}\left(\vec{k}^{\prime}\right)-E_{i}(\vec{k}) \pm \Omega_{\vec{q}, q_{z}}\right)
\end{array}
$$

where $M_{p h}^{i, j}\left(\vec{k}-\vec{k}^{\prime}\right)$ is the matrix element of the deformation potential between polaritons states in different branches $i, j$, given in Ref $\frac{19}{2} . E_{j}\left(\vec{k}^{\prime}\right)$ is the polariton energy in the branch $j$ with wave vector $\vec{k}^{\prime}$ in the two dimensional quantum well plane, $\Omega_{\vec{q}}$ is the acoustic phonon energy with $\vec{Q}=\left(\vec{q}, \vec{q}_{z}\right)$ the total phonon momentum and $n\left(\Omega_{\vec{q}}\right)$ is the Bose occupation number for phonons.

-Electron-polariton relaxation: When polaritons interact with electrons, the experimental and theoretical results show that this mechanism becomes more efficient in the stationary regime. The advantages of including the present relaxation process relies on the fact that low electron density can be tuned easily. It is important to remark that the advantage of this scattering mechanism with respect to the previouly discussed one, is that as the electron has a very small effective mass than an exciton, and the relaxation of bottleneck polaritons to the ground state requires few scattering processes. The matrix elements for the transition rates are calculated as:

$$
\begin{array}{r}
W_{\vec{k}, \vec{k}^{\prime}}^{i, j(e)}=2 \pi \sum_{\vec{k}_{e}}\left|M_{(e l)}^{i, j}\left(\vec{k}-\vec{k}^{\prime}\right)\right|^{2} f_{\vec{k}_{e}}\left(1-f_{\vec{k}_{e}+\vec{k}-\vec{k}^{\prime}}\right) \\
\delta\left(E_{j}\left(\vec{k}^{\prime}\right)-E_{i}(\vec{k})-\frac{1}{2 m_{e}}\left(k_{e}^{2}-\left|\vec{k}_{e}+\vec{k}-\vec{k}^{\prime}\right|^{2}\right)\right)
\end{array}
$$

where $f_{\mathbf{k}_{e}}$ is the Fermi-Dirac distribution function for an $k_{e}$ electron momentum and $M_{(e l)}^{i, j}=V_{d i r} \pm V_{e x c}$ is the matrix element of interaction between an electron and polariton $\stackrel{1}{\underline{1}}$, corresponding to the triplet configuration $(+$, parallel electron spins, $\left.V_{\text {dir }}\right)$, and the single configuration (-, antiparallel electron spins, $\left.V_{\text {exc }}\right)$. In the following, we shall only include the stronger electron-polariton scattering of triplet configuration. The electron Fermi-Dirac distribution is given by $f_{k_{e}}=\left[e^{\left(E_{e}\left(k_{e}\right)-\mu\right) / k_{B} T}+1\right]^{-1}$ where in the two dimensional case the chemical potential is $\mu=k_{B} T \ln \left(e^{E_{f} / K_{B} T}-1\right)$, and we assume that the Fermi energy is given by the free $2 \mathrm{D}$ electrons value: $E_{f}=\pi n_{e} / m_{e}$, $n_{e}$ being the free electrons density. We also assume that the dynamics of free electrons is so fast that we may assume that equilibrium is reached at times shorter than other ones.

\section{NUMERICAL RESULTS}

In order to clarify how is the effectiveness of ph-pol or el-pol, we compare the results for both mechanisms on the relaxation polaritons. The Bolztmann equation 4 includes both electron and phonon relaxation. The details of the $W$ matrix elements have been described in detail in Ref $\stackrel{1}{\underline{1}}$. We present results for the following set of parameters: Well width of $L_{z}=60 \dot{A}$, Rabi splitting $\Omega=4.8 \mathrm{meV}, n=3.07$, electron (hole) deformation potential $D_{e(h)}=12(-7) \mathrm{eV}$, longitudinal sound velocity $u=4.81 \times 10^{3} \mathrm{~m} / \mathrm{s}$, mass density of the solid $\rho=5.3 \times 10^{3} \mathrm{~kg} / \mathrm{m}^{3}$, radiative cavity(exciton) times $\tau_{c(x)}=50(200 \mathrm{ps})$ The decay and generation rates as a function of $k$ are taken from Ref 15 .

In Fig. 1(a), 1(b) and 1(c) we show the two possibilities for relaxation mechanisms. Fig. 1(a) corresponds to the polariton population relaxing by acoustic phonons and in Fig. 1(b) the same but only including el-pol relaxation. Fig. 1(c) includes at the same time both mechanism. We have taken $T=2 K, \delta=\omega_{\text {cav }}-\omega_{e x c}=-1 m e V$. As can be seen, the bottleneck suppression occurs due to the el-pol effects, but it is not lifted completely. In Fig. 1(a) the polariton population reaches its maximum through phonon relaxation for $k \approx 1.5 \times 10^{4} \mathrm{~cm}^{-1}$ while for el-pol relaxation it start to rise at $k \approx 3.2 \times 10^{4} \mathrm{~cm}^{-1}$ as can be seen in Fig. 1(b). The differenice in the position of the 
maximums is a consequence of the restricted phase space filling at this low temperatures, where emission polariton processes are less probable than absorption processes. However, for $t<0.5 n s$, a polariton population grows due to the relaxation by phonons and the relaxation by free carrier electrons starts to grow after 0.7 ns. In Fig. 1(c) both mechanisms are included at the same time. The present results show that for this low temperatures it is impossible to distinguish which of both relaxation mechanisms is relevant to suppress the bottleneck. It is important to remark that the bottleneck is relaxed if the non-resonant power pump is increased and the electron relaxation becomes more inefficient than phonons (not shown).

In order to describe better the experimental data, and to elucidate which of these mechanisms is more important, the lattice temperature is raised to $T=10 \mathrm{~K}$ with the same parameters as in Fig. 1. The numerical data are depicted in Fig. 2(a) (ph-pol), Fig. 2(b) (el-pol), Fig. 2(c) (ph-pol + el-pol). As be expected, the ph-pol scattering allows the bottleneck relaxation to lower $k$ states, but the bottleneck is partially depleted and remains at $k \approx 1.2 \times 10^{4} \mathrm{~cm}^{-1}$, showing a complete range of empty states below this value. However, if the el-pol mechanism is included, the bottleneck relaxes to lower, states showing that it becomes more efficient than ph-pol. In Fig. 2(b) it is possible to see that the el-pol mechanisn becomes more efficient to relax completely the bottleneck and populates the $k=0$ states. When both mechanisms are included, clearly the restriction of the state space is broken and the lower states becomes polariton populated. Our results explicitly show that for lattice temperatures greater than the electronic temperature $\left(k_{B} T_{e}=\pi n_{e} / m_{e}=2 K\right)$ the electron gas becomes the more efficient mechanism to the polariton relaxation. The population reaches the steady state around $t=1.5 \mathrm{~ns}$, and after this time the phonon relaxation becomes more inefficient.

In Figs. 3. we show the polariton population distribution for different times. Fig. 3(a) shows that for $T=2 K$ the populations follow a Boltzmann distribution and for both relaxation mechanisms the steady state is obtained at $t \approx 2.5 n s$. Fig. 3(b) includes both mechanism showing that el-pol relaxation is not efficient enough. Moreover, if the temperature is increased, $T=10 \mathrm{~K}$, in Fig. 3(c) the polariton population maximum peak is exchanged with respect to Fig. 3(a) and lower $k$ states become populated, showing clearly an asymmetric behavior for the polariton population with respect to the $k$-value where the population takes its maximum. Clearly, for low excitation, the shape of the population develops a symmetric shape for low temperatures, but it becomes deformed when the temperature is increased, demonstrating clearly that the main important relaxation mechanism for the bottleneck comes from

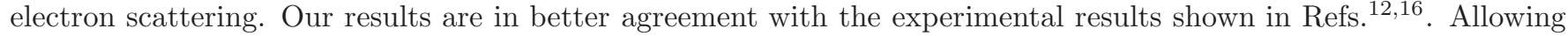
the el-pol scattering, the bottleneck is shifted towards lower momenta, changing the population in an asymmetric way compared with the pnonon-polariton relaxation.

In summary, we have used a model of bosonic particles with a strong electron-polariton and phonon-polariton coupling, and have shown numerically exact results for the population relaxation as a function of momentum and time. The competition between the itinerancy of the electron and the phonon interaction gives rise to a changeover of the behavior of the polariton population from low temperatures to high temperatures in which we elucidate which one of these two mechanisms is more important in order to relax the bottleneck. Our results demonstrate clearly that Doan's 15 theoretical results are valid for very low temperatures and explain scarcely the experimental data. Our results, additionally show that there exists a fundamental difference between a relaxation involving electron or acoustical phonon elastic scattering with polaritons. We have clearly shown that the electron-polariton mechanism becomes more efficient for $t \approx 2 n s$ and it is the responsible for the relaxation in the stationary limit, leading to a huge population at $k=0$ values.

The authors acknowledge to L. Quiroga and A. Reyes for fruitful discussions. Financial support from Facultad de Ciencias, Universidad de los Andes, Banco de la República and COLCIENCIAS:1204-05-11408 are gratefully acknowledged.

1 P.G. Savvidis, J.J. Baumberg, R.M. Stevenson, M.S. Skolnick, D.M. Whittaker, J.S. Roberts, Phys. Rev. Lett. 84 (2000) 1547.

${ }^{2}$ P.G Lagoudakis, P. G. Savvidis, J. J. Baumberg, D. M. Whittaker, P. R. Eastham, M. S. Skolnick, J. S. Roberts, Phys. Rev. B. 65 (2002) 161310 .

${ }^{3}$ R.M. Stevenson, V.N. Astratov, M.S. Skolnick, D.M. Whittaker, M. Emam-Ismail, A.I. Tartakovskii, P.G. Savvidis, J.J. Baumberg, J.S. Roberts, Phys. Rev. Lett. 85 (2000) 3680.

4 J.J. Baumberg, P. G. Savvidis, R. M. Stevenson, A. I. Tartakovskii, M. S. Skolnick, D. M. Whittaker, J. S. Roberts, Phys. Rev. B 62 (2000) 16247.

${ }^{5}$ C. Ciuti, P. Schwendimann, B. Deveaud, A. Quattropani, Phys. Rev. B 62 (2000) 4825.

6 Y. Rubo, F.P. Laussy, A. Kavokin, P. Bigenwald, Phys. Rev. Lett. 91 (2003) 156403.

7 H. T. Cao, T. D. Doan, D. B. Tran Thoai, Phys. Rev. B 69, (2004) 245325.

${ }^{8}$ P. Senellart, J. Bloch, B. Sermage, J. Y. Marzin, Phys. Rev. B 62, (2000) 16263. 

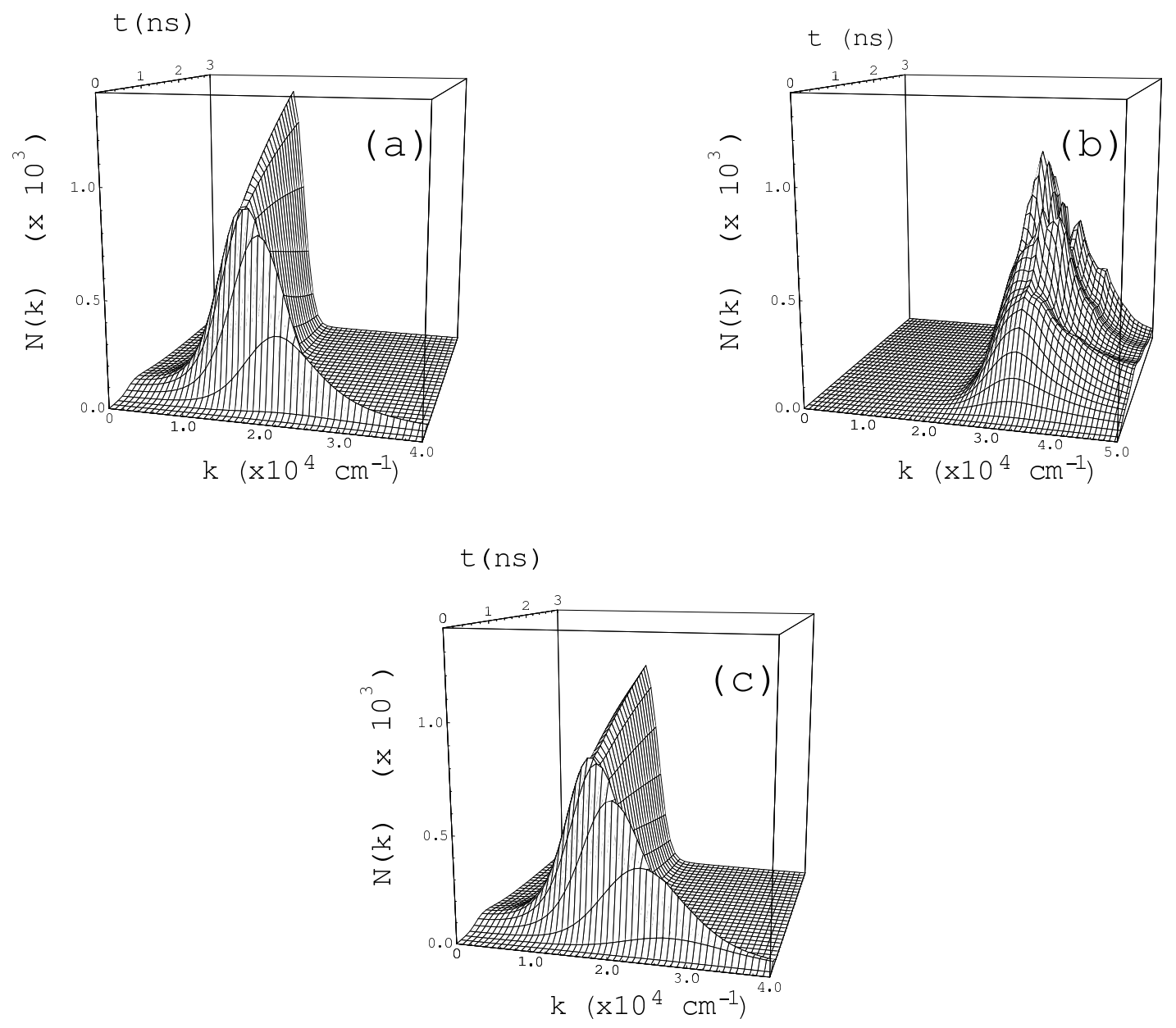

FIG. 1: Distribution function for different interaction mechanisms with $n_{x}=8 \times 10^{9} \mathrm{~cm}^{-2} . T=2 \mathrm{~K}, \delta=-1 \mathrm{meV}$ and the pump laser is non-resonant applied at $k=6 \times 10^{4} \mathrm{~cm}^{-1}$. a) Only ph-pol relaxation; b) Only el-pol relaxation; c) Both mechanism includes at the same time

9 H. Huang, F. Tassone, Y. Yamamoto, Phys. Rev. B 61 (2000) R7854.

10 P.G Lagoudakis, M. D. Martin, J. J. Baumberg, A. Qarry, E. Cohen, and L. N. Pfeiffer, Phys. Rev. Lett. 90, (2003) 206401.

11 G. Malpuech, A. Kavokin, A. Di Carlo, J. J. Baumberg, Phys. Rev. B 65, (2002) 153310.

12 R. Butté, G. Delalleau, A. I. Tartakovskii, M. S. Skolnick, V. N. Astratov, J. J. Baumberg, G. Malpuech, A. Di Carlo, A. V. Kavokin, and J. S. Roberts, Phys. Rev. B 65 (2002) 205310.

13 B. Sermage, S. Long, I. Abram, J. Y. Marzin, J. Bloch, R. Planel, V. ThierryMieg, Phys. Rev. B 53, (1996) 16516.

14 P.G. Savvidis, J.J. Baumberg, R.M. Stevenson, M.S. Skolnick, D.M. Whittaker, J.S. Roberts, Phys. Rev. B 62 (2000) R13278.

15 T. D. Doan and D. B. Tran Thoai, Solid State Commun. 123, (2002) 427.

16 A. I. Tartakovskii, D. N. Krizhanovskii, G. Malpuech, M. Emam-Ismail, A. V. Chernenko, A. V. Kavokin, V. D. Kulakovskii, M. S. Skolnick, and J. S. Roberts Phys. Rev. B 67 (2003) 165302.

17 D. Porras, C. Ciuti, J. J. Baumberg, C. Tejedor, Phys. Rev. B 66, (2002) 085304.

18 T. D. Doan, Huy Thien Cao, D. B. Tran Thoai, Phys. Rev. B 72, (2005) 085301.

19 F. Tassone, C. Piermarocchi, V. Savona, A. Quattropani, Phys. Rev. B 53 (1996) 7642. 
$t(n s)$

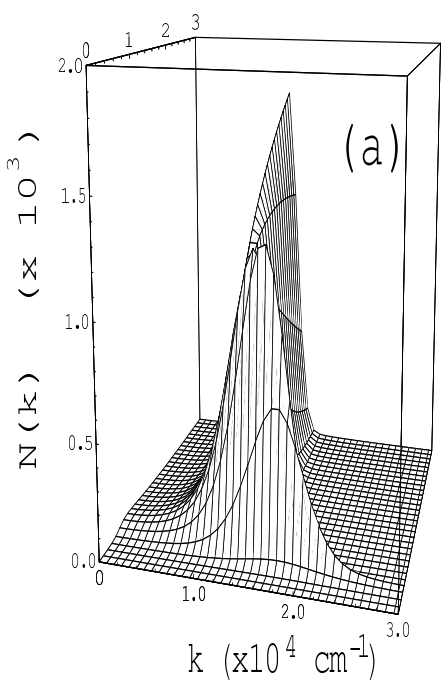

t (ns)

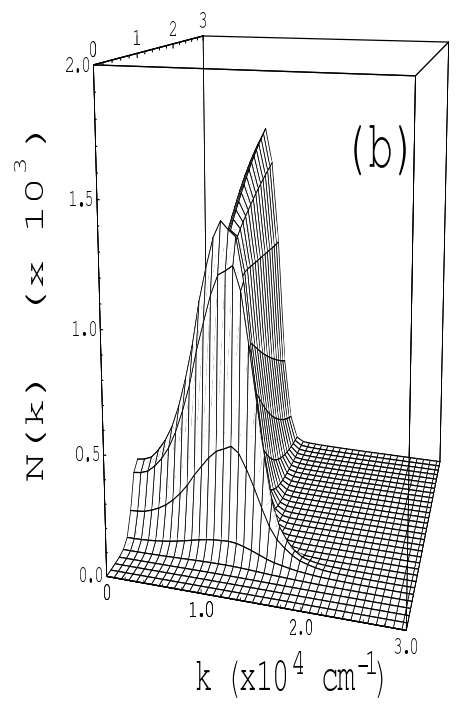

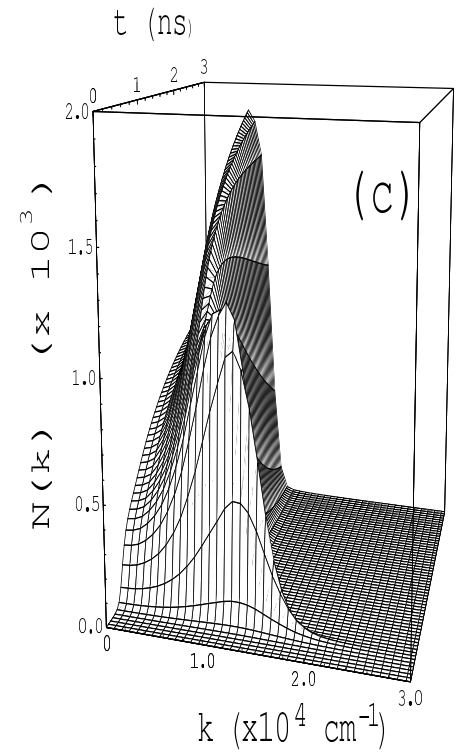

FIG. 2: Distribution function $N_{k}(t)$ with $T=10 \mathrm{~K}, n_{x}=8 \times 10^{9} \mathrm{~cm}^{-2}$ and $\delta=-1 \mathrm{meV}$ for a) Only ph-pol interaction mechanism, b) Only el-pol interaction mechanism, c) ph-pol + el-pol interaction mechanisms. 

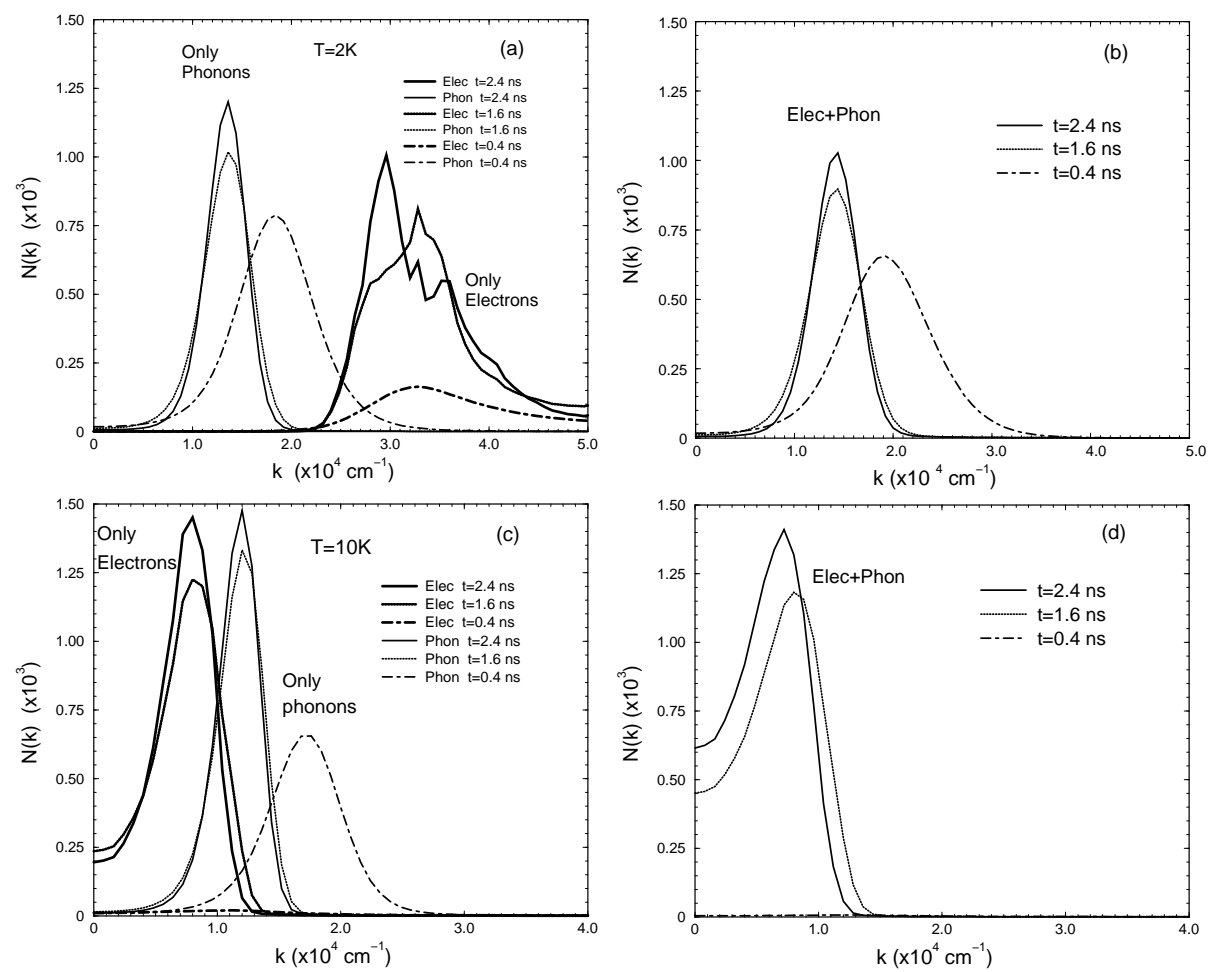

FIG. 3: Comparision of the time evolution of the polariton population at $\mathrm{T}=2 \mathrm{~K}$ relaxating through (a) phonons and electrons, alone each one and (b) the sum of both processes. (c) and (d) the same as (a) and (b) but for T=10 K up to $2.4 \mathrm{~ns}$ 